\title{
Application of Fixed-Point Index Theory for a Nonlinear Fractional Boundary Value Problem with an Advanced Argument
}

\author{
Li Wu ${ }^{1}$ and Chuanzhi Bai ${ }^{2}{ }^{2}$ \\ ${ }^{1}$ Department of Mathematics, Nanjing Institute of Technology, Nanjing, Jiangsu 211167, China \\ ${ }^{2}$ Department of Mathematics, Huaiyin Normal University, Huai'an, Jiangsu 223300, China
}

Correspondence should be addressed to Chuanzhi Bai; czbai@hytc.edu.cn

Received 20 July 2021; Accepted 8 September 2021; Published 24 September 2021

Academic Editor: Bo Yang

Copyright (c) $2021 \mathrm{Li} \mathrm{Wu}$ and Chuanzhi Bai. This is an open access article distributed under the Creative Commons Attribution License, which permits unrestricted use, distribution, and reproduction in any medium, provided the original work is properly cited.

In this paper, we investigate the existence of positive solutions of a class of fractional three-point boundary value problem with an advanced argument by using fixed-point index theory. Our results improve and extend some known results in the literature. Two examples are given to demonstrate the effectiveness of our results.

\section{Introduction}

Three-point boundary value problems arise in a variety of different applied mathematics and physics. In [1], Gupta studied the solvability of three-point BVPs for nonlinear second-order ordinary differential equations. Since then, many authors have investigated the existence and multiplicity of solutions for three-point boundary value problems for nonlinear integer-order ordinary differential equations and nonlinear fractional-order differential equations, see [2-19] and the references therein.

In [2], Ma considered the following three-point boundary value problem:

$$
\begin{cases}u^{\prime \prime}(t)+a(t) f(u)=0, & 0<t<1 \\ u(0)=0, & u(1)=\alpha u(\eta)\end{cases}
$$

where $\alpha>0, \eta \in(0,1), \alpha \eta<1, a \in C([0,1], t[0, \infty))$, and $f \in C([0, \infty), t[0, \infty))$ is suplinear or sublinear. The existence of at least one positive solution for BVP (1) is obtained by virtue of the Krasnosel'skii fixed-point theorem.

In [3], due to the well-known Guo-Krasnoselskii fixedpoint theorem [20], Wang et al. proved the existence of a positive solution to the following three-point BVPs of nonlinear fractional-order differential equation with an advanced argument:

$$
\begin{cases}{ }^{C} D^{\alpha} u(t)+a(t) f(u(\theta(t)))=0, & 0<t<1, \\ u(0)=u^{\prime \prime}(0)=0, & u(1)=\beta u(\eta),\end{cases}
$$

where $2<\alpha \leq 3, \eta \in(0,1), 0<\beta<(1 / \eta),{ }^{C} D^{\alpha}$ is the Caputo fractional derivative, and $f:[0, \infty) \longrightarrow[0, \infty)$ is a continuous function.

Recently, in [4], Pourhadi et al. considered the following three-point boundary value problem:

$$
\begin{cases}{ }^{C} D^{\alpha} x(t)=f\left(t, x(t), x^{\prime}(t)\right), & a<t<b, 1<\alpha<2, \\ x(a)=0, x(b)=\mu x(\eta), & a<\eta<b, \mu>\lambda,\end{cases}
$$

where $\lambda=(b-a) /(\eta-a),{ }_{a}^{C} D^{\alpha}$ stands for the Caputo fractional derivative, and $f:[a, b] \times \mathbb{R} \times \mathbb{R} \longrightarrow \mathbb{R}$ is a continuous function. The existence of positive solutions for BVPs (3) is given by using the inequalities related with Green's function and two well-known fixed-point theorems.

Motivated by the mentioned works, in this paper, we consider the following fractional-order three-point boundary value problem: 


$$
\begin{cases}{ }^{C} D^{\alpha} u(t)+a(t) f(u(\theta(t)))=0, & 0<t<1, \\ u(0)-\beta u^{\prime}(0)=u^{\prime \prime}(0)=0, & u(1)=\gamma u(\eta),\end{cases}
$$

where $2<\alpha \leq 3, \quad 0<\eta<1, \quad \beta \geq 0, \quad 0<\gamma<((1+\beta) /(\beta+\eta))$, ${ }^{C} D^{\alpha}$ is the Caputo fractional derivative, $a \in C([0,1], t$ $[0, \infty))$, and $f \in C^{1}[[0, \infty), t[0, \infty))$.

Based on the fixed-point index theory, the existence of at least one positive solution to BVP (4) has been obtained. The main results of the paper by Wang et al. [3] have been extended and improved.

Remark 1. If $\beta=0$, then the fractional differential equations under boundary value conditions (4) reduces to the BVPs (2).

The paper is organized in this order. In Section 2, we present basic definitions on fractional calculus theory and some lemmas which play an important role in the proof of our main results. In Section 3, under an adequate condition imposed on $f$, we prove the existence of positive solutions of BVPs (4). In Section 4, two examples are given to demonstrate our results.

\section{Preliminaries}

For convenience, some fundamental definitions on fractional calculus are given. For more details, we refer to [21].

Definition 1. Let $\alpha \geq 0$ and $f$ be a real function defined on $[a, b]$. The Riemann-Liouville fractional integral of order $\alpha$ is defined by $I^{0} f \equiv f$ and

$$
\left(I^{\alpha} f\right)(t)=\frac{1}{\Gamma(\alpha)} \int_{0}^{t}(t-s)^{\alpha-1} f(s) \mathrm{d} s, \quad \alpha>0, t \in[0,1] .
$$

Definition 2. The Caputo derivative of fractional order $\alpha \geq 0$ is defined by ${ }^{C} D^{0} f \equiv f$ and

$$
\left({ }^{C} D^{\alpha} f\right)(t)=\frac{1}{\Gamma(n-\alpha)} \int_{0}^{t}(t-s)^{n-\alpha-1} f^{(n)}(s) \mathrm{d} s, \quad \alpha>0, t \in[0,1],
$$

where $n$ is the smallest integer greater or equal to $\alpha$. In the following, we state some properties of fractional operators that will be used in proving our main results.

Lemma 1 (see [21]). Let $\alpha, \beta \in \mathbb{R}^{+}$and $u \in L_{1}[0,1]$; then, $I^{\alpha} I^{\beta} u(x)=I^{\alpha+\beta} u(x)$ holds almost everywhere on $[0,1]$.

Lemma 2 (see [21]). Let $\alpha \in \mathbb{R}^{+}$and $u \in C[0,1]$; then, ${ }^{C} D^{\alpha} I^{\alpha} u(x)=u(x)$.

Lemma 3 (see [21]). If $\alpha>0, n$ is the smallest integer greater than or equal to $\alpha$, and $u \in A C^{n}[0,1]$, then

$$
I^{\alpha C} D^{\alpha} u(x)=u(x)+c_{0}+c_{1} t+\cdots+c_{n-1} t^{n-1},
$$

for some $c_{i} \in \mathbb{R}, i=0,1, \ldots, n-1$.

Lemma 4. Let $2<\alpha \leq 3$ and $g \in C^{1}[0,1]$. A function $u$ is $a$ solution of the following boundary value problem:

$$
\begin{aligned}
{ }^{C} D^{\alpha} u(t)+g(t) & =0, \quad 0<t<1, \\
u(0)-\beta u^{\prime}(0) & =u^{\prime \prime}(0)=0, \\
u(1) & =\gamma u(\eta),
\end{aligned}
$$

if and only if $u$ satisfies the integral equation

$$
u(t)=\int_{0}^{1} G(t, s) g(s) \mathrm{d} s,
$$

where

$$
G(t, s)= \begin{cases}\frac{(t+\beta)(1-s)^{\alpha-1}-(t+\beta) \gamma(\eta-s)^{\alpha-1}-(t-s)^{\alpha-1}(1+\beta-(\beta+\gamma) \eta)}{\Gamma(\alpha)(1+\beta-(\beta+\eta) \gamma)}, & 0 \leq s \leq t \leq 1, s \leq \eta, \\ \frac{(t+\beta)(1-s)^{\alpha-1}-(t-s)^{\alpha-1}(1+\beta-(\beta+\gamma) \eta)}{\Gamma(\alpha)(1+\beta-(\beta+\eta) \gamma)}, & 0<\eta \leq s \leq t \leq 1, \\ \frac{(t+\beta)(1-s)^{\alpha-1}-(t+\beta) \gamma(\eta-s)^{\alpha-1}}{\Gamma(\alpha)(1+\beta-(\beta+\eta) \gamma)}, & 0 \leq t \leq s \leq \eta<1, \\ \frac{(t+\beta)(1-s)^{\alpha-1}}{\Gamma(\alpha)(1+\beta-(\beta+\eta) \gamma)} & 0 \leq t \leq s \leq 1, \eta \leq s,\end{cases}
$$

is the Green function of BVP (8)-(9).

$$
u(t)=c_{1}+c_{2} t+c_{3} t^{2}-\int_{0}^{t} \frac{(t-s)^{\alpha-1}}{\Gamma(\alpha)} g(s) \mathrm{d} s
$$

Proof. In view of Lemma 3, we may reduce (8) to an equivalent integral equation:
According to Lemmas 1 and 2, we obtain 


$$
\begin{aligned}
& u^{\prime}(t)=c_{2}+2 c_{3} t-\int_{0}^{t} \frac{(t-s)^{\alpha-2}}{\Gamma(\alpha-1)} g(s) \mathrm{d} s, \\
& u^{\prime \prime}(t)=2 c_{3}-\int_{0}^{t} \frac{(t-s)^{\alpha-3}}{\Gamma(\alpha-2)} g(s) \mathrm{d} s .
\end{aligned}
$$

From $u^{\prime \prime}(0)=0$, we know that $c_{3}=0$. By $u(0)-\beta u^{\prime}(0)=0$, it follows that $c_{1}=\beta c_{2}$. Using the boundary condition $u(1)=\gamma u(\eta)$, we have

$$
\begin{gathered}
c_{2}=-\frac{1}{1+\beta-(\beta+\eta) \gamma} \int_{0}^{1} \frac{(1-s)^{\alpha-1}}{\Gamma(\alpha)} g(s) \mathrm{d} s \\
+\frac{\gamma}{1+\beta-(\beta+\eta) \gamma} \int_{0}^{\eta} \frac{(\eta-s)^{\alpha-1}}{\Gamma(\alpha)} g(s) \mathrm{d} s .
\end{gathered}
$$

Therefore, the solution of BVP (8)-(9) is

$$
\begin{aligned}
u(t)= & -\int_{0}^{t} \frac{(t-s)^{\alpha-1}}{\Gamma(\alpha)} g(s) \mathrm{d} s+\frac{t+\beta}{1+\beta-(\beta+\eta) \gamma} \int_{0}^{1} \frac{(1-s)^{\alpha-1}}{\Gamma(\alpha)} g(s) \mathrm{d} s \\
& -\frac{\gamma(t+\beta)}{1+\beta-(\beta+\eta) \gamma} \int_{0}^{\eta} \frac{(\eta-s)^{\alpha-1}}{\Gamma(\alpha)} g(s) \mathrm{d} s \\
= & \int_{0}^{1} G(t, s) g(s) \mathrm{d} s
\end{aligned}
$$

where the Green function $G(t, s)$ is defined by (11).

Remark 2. For Caputo-type fractional problems, Cichon and Salem in [22] studied the problem of the lack of equivalent between differential and integral forms and pointed out that many papers contain an error in the proof of the equivalent of the fractional-type differential problems and the corresponding integral forms. If $g \in C^{1}[0,1]$, then $g$ is absolutely continuous on $[0,1]$, and we know from [22] that (8) and (12) are equivalent.
Lemma 5. The Green function $G(t, s)$, defined as in Lemma 4, has the following properties:

(i) $G(t, s)$ is a continuous function.

(ii)

$$
G(t, s) \geq H(t, s)= \begin{cases}\frac{(t+\beta) s \gamma \eta^{2-\alpha}(\alpha-1)(1-\eta)(\eta-s)^{\alpha-2}}{\Gamma(\alpha)(1+\beta-(\beta+\eta) \gamma)}, & 0 \leq s \leq t \leq 1, s \leq \eta, \\ \frac{(t+\beta) \gamma \eta(1-s)^{\alpha-1}}{\Gamma(\alpha)(1+\beta-(\beta+\eta) \gamma)}, & 0<\eta \leq s \leq t \leq 1, \\ \frac{(t+\beta)(1-\gamma \eta)(1-s)^{\alpha-1}}{\Gamma(\alpha)(1+\beta-(\beta+\eta) \gamma)}, & 0 \leq t \leq s \leq \eta<1, \\ \frac{(t+\beta)(1-s)^{\alpha-1}}{\Gamma(\alpha)(1+\beta-(\beta+\eta) \gamma)}, & 0 \leq t \leq s \leq 1, \eta \leq s .\end{cases}
$$

Proof. It is obvious that (i) holds by the expression of $G(t, s)$. Now, we prove that (ii) holds. When $t=0$, $G(0, s)=0$. When $t \neq 0$, we show three different cases.

Case 1. For $0 \leq s \leq t \leq 1, s<\eta$, let

$$
\begin{aligned}
g_{1}(t, s)= & (t+\beta)(1-s)^{\alpha-1}-(t+\beta) \gamma(\eta-s)^{\alpha-1} \\
& -(t-s)^{\alpha-1}(1+\beta-(\beta+\eta) \gamma) .
\end{aligned}
$$

Then, by Remark 3,

$$
\begin{aligned}
g_{1}(t, s)= & (t+\beta)(1-s)^{\alpha-1}-(t+\beta) \gamma \eta^{\alpha-1}\left(1-\frac{s}{\eta}\right)^{\alpha-1} \\
& -t^{\alpha-1}\left(1-\frac{s}{t}\right)^{\alpha-1}(1+\beta-(\beta+\eta) \gamma)
\end{aligned}
$$




$$
\begin{aligned}
& \geq(t+\beta)(1-s)^{\alpha-1}-(t+\beta) \gamma \eta^{\alpha-1}\left(1-\frac{s}{\eta}\right)^{\alpha-1} \\
& -t^{\alpha-1}(1-s)^{\alpha-1}(1+\beta-(\beta+\eta) \gamma) \\
& \geq(t+\beta)(1-s)^{\alpha-1}-(t+\beta) \gamma \eta\left(1-\frac{s}{\eta}\right)^{\alpha-1} \\
& -t(1-s)^{\alpha-1}(1+\beta-(\beta+\eta) \gamma) \\
& =(1-s)^{\alpha-1}[(t+\beta)-t(1+\beta-(\beta+\eta) \gamma)] \\
& -(t+\beta) \gamma \eta\left(1-\frac{s}{\eta}\right)^{\alpha-1} \\
& =(1-s)^{\alpha-1}[t \eta \gamma+\beta(1-t+t \gamma)]-(t+\beta) \gamma \eta\left(1-\frac{s}{\eta}\right)^{\alpha-1} \\
& \geq(1-s)^{\alpha-1}(t \eta \gamma+\beta \gamma \eta)-(t+\beta) \gamma \eta\left(1-\frac{s}{\eta}\right)^{\alpha-1} \\
& =(t+\beta) \gamma \eta\left[(1-s)^{\alpha-1}-\left(1-\frac{s}{\eta}\right)^{\alpha-1}\right] \\
& =(t+\beta) \gamma \eta(\alpha-1) \int_{1-(s / \eta)}^{1-s} x^{\alpha-2} \mathrm{~d} x \\
& \geq(t+\beta) \gamma \eta(\alpha-1)\left(1-\frac{s}{\eta}\right)^{\alpha-2}\left[(1-s)-\left(1-\frac{s}{\eta}\right)\right] \\
& =(t+\beta) s \gamma \eta^{2-\alpha}(\alpha-1)(1-\eta)(\eta-s)^{\alpha-2} \text {. }
\end{aligned}
$$

Case 2. For $0<\eta \leq s \leq t \leq 1$, let

$$
g_{2}(t, s)=(t+\beta)(1-s)^{\alpha-1}-(t-s)^{\alpha-1}(1+\beta-(\beta+\eta) \gamma) .
$$

Then, by Remark 3,

$$
\begin{aligned}
g_{2}(t, s) & =(t+\beta)(1-s)^{\alpha-1}-t^{\alpha-1}\left(1-\frac{s}{t}\right)^{\alpha-1}(1+\beta-(\beta+\eta) \gamma) \\
& \geq(t+\beta)(1-s)^{\alpha-1}-t(1-s)^{\alpha-1}(1+\beta-(\beta+\eta) \gamma) \\
& =(1-s)^{\alpha-1}[(t+\beta)-t(1+\beta-(\beta+\eta) \gamma)] \\
& \geq(t+\beta) \gamma \eta(1-s)^{\alpha-1} .
\end{aligned}
$$

Case 3. For $0 \leq t \leq s \leq \eta<1$, let

$$
g_{3}(t, s)=(t+\beta)(1-s)^{\alpha-1}-(t+\beta) \gamma(\eta-s)^{\alpha-1} .
$$

Then,

$$
\begin{aligned}
g_{3}(t, s) & =(t+\beta)\left[(1-s)^{\alpha-1}-\gamma \eta^{\alpha-1}\left(1-\frac{s}{\eta}\right)^{\alpha-1}\right] \\
& \geq(t+\beta)\left[(1-s)^{\alpha-1}-\gamma \eta^{\alpha-1}(1-s)^{\alpha-1}\right] \\
& \geq(t+\beta)(1-\gamma \eta)(1-s)^{\alpha-1} .
\end{aligned}
$$

The proof is complete.
Remark 3. We have inequality (18) by using $1-t+t \gamma \geq \gamma \eta$. In fact, if $\gamma \geq 1$, since $0<\gamma<((1+\beta) /(\beta+\eta))$ that $1-\gamma \eta>\beta(\gamma-1) \geq 0 \geq t(1-\gamma)$, which implies that $1-t+t \gamma \geq \gamma \eta$. If $0<\gamma<1$, then $1-\gamma \eta>1-\gamma \geq t(1-\gamma)$, which also yields that $1-t+t \gamma \geq \gamma \eta$.

Remark 4. From Lemma 5, we know that $G(t, s) \geq H(t, s) \geq 0$, for all $t, s \in[0,1]$.

Lemma 6. Let $2<\alpha \leq 3$ and $0<\gamma<((1+\beta) /(\beta+\eta))$. Assume $g \in C^{1}([0,1], t[0, \infty))$; then, the unique solution $u$ of (8)-(9) satisfies $u(t) \geq 0$ for any $t \in[0,1]$. Moreover, the unique solution $u$ of (8)-(9) satisfies

$$
\inf _{t \in[\eta, 1]} u(t) \geq \delta\|u\|
$$

where $\delta=\min \{\gamma \eta,(\gamma(1-\eta) /(1-\gamma \eta)), \eta\}$.

Proof. By Lemmas 4 and 5 and Remark 4, we know that $u(t) \geq 0, \forall t \in[0,1]$. From Lemma 4 , one has

$$
u^{\prime \prime}(t)=-\int_{0}^{t} \frac{(t-s)^{\alpha-3}}{\Gamma(\alpha-2)} g(s) \mathrm{d} s \leq 0 .
$$

By applying the concavity of $u$, similar to the proof of Lemma 4 in [2], we can obtain that (23) holds.

Lemma 7 (see [23]). Let $P$ be a reproducing cone in a real Banach space $E$ and $L: E \longrightarrow E$ be a compact linear operator with $L(P) \subset P$ and spectral radius $r(L)$. If $r(L)>0$, then there exists $\varphi \in P \backslash\{0\}$ such that $L \varphi=r(L) \varphi$.

Theorem 1 (see [23]). Assume that $E$ is a Banach space, $P \subset E$ is a cone, and $\Omega(P)$ is a bounded open subset in $P$. Moreover, assume that $T: \overline{\Omega(P)} \longrightarrow P$ is a completely continuous operator. Then, the following conclusions hold:

(i) If there exists $u_{0} \in P \backslash\{0\}$ such that $u \neq T u+\lambda u_{0}$, for all $u \in \partial \Omega(P)$ and $\lambda \geq 0$, then the fixed-point index $i(T, \Omega(P), P)=0$

(ii) If $0 \in \Omega(P)$ and $T u \neq \lambda u$, for all $u \in \partial \Omega(P)$ and $\lambda \geq 1$, then the fixed-point index $i(T, \Omega(P), P)=1$

\section{Main Results}

Let $E=C[0,1]$ be the Banach space endowed with the sup norm and define the cone:

$$
P=\left\{x \in E: \quad x \geq 0, \inf _{t \in[\eta, 1]} x(t) \geq \delta\|x\|\right\},
$$

where $\delta$ is defined in Lemma 6. Define the linear operator $L: P \longrightarrow P$ as follows:

$$
L u(t)=\int_{0}^{1} G(t, s) a(s) u(\theta(s)) \mathrm{d} s .
$$

Lemma 8. Assume that

(H1) $a \in C^{1}([0,1], t[0, \infty))$ and $a$ does not vanish identically on any subinterval 
(H2) The advanced argument $\theta \in C((0,1),(0,1))$ and $t \leq \theta(t) \leq 1, \forall t \in(0,1)$

hold. Then, the spectral radius of the operator $L$ is positive, that is, $r(L)>0$.

Proof. Taking $u(t)=t^{\alpha-1}$, then $\|u\|=1$. By $(H 1),(H 2)$, and Lemma 5, we have

$$
\begin{aligned}
L u(t) & =\int_{0}^{1} G(t, s) a(s) u(\theta(s)) \mathrm{d} s \geq \int_{0}^{1} H(t, s) a(s) \theta(s)^{\alpha-1} \mathrm{~d} s \\
& \geq \int_{0}^{1} H(t, s) a(s) s^{\alpha-1} \mathrm{~d} s \\
& \geq \int_{0}^{\eta} \frac{(t+\beta) s \gamma \eta^{2-\alpha}(\alpha-1)(1-\eta)(\eta-s)^{\alpha-2}}{\Gamma(\alpha)(1+\beta-(\beta+\eta) \gamma)} a(s) s^{\alpha-1} \mathrm{~d} s \\
& \geq t^{\alpha-1} \frac{\gamma \eta^{2-\alpha}(\alpha-1)(1-\eta)}{\Gamma(\alpha)(1+\beta-(\beta+\eta) \gamma)} \int_{0}^{\eta} a(s) s^{\alpha}(\eta-s)^{\alpha-2} \mathrm{~d} s \\
& :=\omega t^{\alpha-1}>0 .
\end{aligned}
$$

Since $L: P \longrightarrow P$, in view of the monotonicity of $L$, we obtain

$$
L^{2} u(t)=L(L u(t))>L\left(\omega t^{\alpha-1}\right)>\omega L\left(t^{\alpha-1}\right)>\omega^{2} t^{\alpha-1} .
$$

Repeating the process gives $L^{n} u(t)>\omega^{n} t^{\alpha-1}$. Hence, we get $\left\|L^{n}\right\|>\omega^{n}$. So,

$$
\begin{aligned}
\left\|L^{n}\right\|^{1 / n} & >\omega, \\
r(L) & =\lim _{n \longrightarrow \infty}\left\|L^{n}\right\|^{1 / n}>\omega>0 .
\end{aligned}
$$

The proof is completed.

Define the operator $T: P \longrightarrow E$ by

$$
(\mathrm{Tu})(t)=\int_{0}^{1} G(t, s) a(s) f(u(\theta(s))) \mathrm{d} s, \quad 0 \leq t \leq 1 .
$$

For convenience, set

$$
\begin{aligned}
f_{0} & =\lim _{u \longrightarrow 0^{+}} \frac{f(u)}{u}, \\
f_{\infty} & =\lim _{u \longrightarrow+\infty} \frac{f(u)}{u}, \\
P_{c} & =\{u \in P: \quad\|u\|<c\} .
\end{aligned}
$$

Lemma 9. Let $f \in C^{1}([0, \infty), t[0, \infty))$. Assume that (H1) and (H2) hold and $0 \leq f_{0}<(1 / r(L))$. Then, there exists $\rho_{0}>0$ such that, for $\rho \in\left(0, \rho_{0}\right], i\left(T, P_{\rho}, P\right)=1$.

Proof. From Lemma 6, we know that $T P \subset P$. It is easy to check that the operator $T: P \longrightarrow P$ is completely continuous. It follows from $f_{0}<(1 / r(L))$ that there exist $\varepsilon>0$ and $\rho_{0}>0$ such that, for $0 \leq u \leq \rho_{0}$,

$$
f(u) \leq\left(\frac{1}{r(L)}-\varepsilon\right) u .
$$

For $0<\rho<\rho_{0}$, we will show that $\lambda u \neq T u$, for all $u \in \partial P_{\rho}$ and $\lambda \geq 1$. Otherwise, there exist $u_{0} \in \partial P_{\rho}$ and $\lambda_{0} \geq 1$ such that

$$
T u_{0}=\lambda_{0} u_{0}
$$

By (32), one has

$$
\begin{aligned}
\lambda_{0} u_{0}(t)=T u_{0}(t) & =\int_{0}^{1} G(t, s) a(s) f\left(u_{0}(\theta(s))\right) \mathrm{d} s \\
& \leq\left(\frac{1}{r(L)}-\varepsilon\right) L u_{0}(t),
\end{aligned}
$$

that is,

$$
\begin{aligned}
u_{0}(t) & \leq \frac{(1 / r(L))-\varepsilon}{\lambda_{0}} L u_{0}(t) \leq\left(\frac{(1 / r(L))-\varepsilon}{\lambda_{0}}\right)^{2} L^{2} u_{0}(t) \\
& \leq \cdots \leq\left(\frac{(1 / r(L))-\varepsilon}{\lambda_{0}}\right)^{n} L^{n} u_{0}(t) \leq\left(\frac{(1 / r(L))-\varepsilon}{\lambda_{0}}\right)^{n}\left\|L^{n}\right\|\left\|u_{0}\right\| .
\end{aligned}
$$

Thus,

$$
\left\|u_{0}\right\| \leq\left(\frac{(1 / r(L))-\varepsilon}{\lambda_{0}}\right)^{n}\left\|L^{n}\right\|\left\|u_{0}\right\|,
$$

which implies that

$$
\frac{\lambda_{0}}{(1 / r(L))-\varepsilon} \leq\left\|L^{n}\right\|^{1 / n} \longrightarrow r(L), \quad n \longrightarrow \infty
$$

It means that

$$
1 \leq \lambda_{0} \leq 1-\varepsilon r(L) .
$$

This is a contradiction. By Theorem 1 (ii), we obtain that $i\left(T, P_{\rho}, P\right)=1$, for each $\rho \in\left(0, \rho_{0}\right]$. The proof is completed.

Lemma 10. Let $f \in C^{1}([0, \infty), t[0, \infty))$. Assume that (H1) and (H2) hold and $(1 / r(L))<f_{\infty} \leq \infty$. Then, there exists $\tau_{0}>0$ such that, for $\tau>\tau_{0}, i\left(T, P_{\tau}, P\right)=0$.

Proof. Since $f_{\infty}>(1 / r(L))$, there exist $\varepsilon>0$ and $\tau_{0}>0$ such that, for $u>\tau_{0}$,

$$
f(u) \geq\left(\frac{1}{r(L)}+\varepsilon\right) u .
$$

For $\tau>\tau_{0}$, assume that $u \neq T u$ and $u \in \partial P_{\tau}$. By Lemma 7 and Theorem 1 (i), we need only to prove that

$$
u \neq T u+\lambda \varphi, \quad \lambda>0,
$$

where $\varphi \in P \backslash\{0\}$ with $L \varphi=r(L) \varphi$. Otherwise, there exist $u_{0} \in \partial P_{\tau}$ and $\lambda_{0}>0$ such that

$$
u_{0}=T u_{0}+\lambda_{0} \varphi,
$$

which implies that $u_{0} \geq T u_{0}$ and $u_{0} \geq \lambda_{0} \varphi$. So, by (39), we obtain that 


$$
\begin{aligned}
T u_{0}(t) & =\int_{0}^{1} G(t, s) a(s) f\left(u_{0}(\theta(s))\right) \mathrm{d} s \geq\left(\frac{1}{r(L)}+\varepsilon\right) L u_{0}(t) \geq\left(\frac{1}{r(L)}+\varepsilon\right) \lambda_{0} L \varphi(t) \\
& =\left(\frac{1}{r(L)}+\varepsilon\right) \lambda_{0} r(L) \varphi(t)>\lambda_{0} \varphi(t),
\end{aligned}
$$

by $((1 / r(L))+\varepsilon) r(L)>1$. Together with (41), one has $u_{0} \geq 2 \lambda_{0} \varphi$. From (42), we have that $T u_{0} \geq 2 \lambda_{0} \varphi$. Thus, $u_{0} \geq 3 \lambda_{0} \varphi$. Repeating this process, we get that $u_{0} \geq n \lambda_{0} \varphi$; this means that $\left\|u_{0}\right\| \geq n \lambda_{0}\|\varphi\| \longrightarrow \infty$, as $n \longrightarrow \infty$. This is a contradiction. It follows from Theorem 1 (i) that $i\left(T, P_{\tau}, P\right)=0$, for each $\tau>\tau_{0}$. The proof is completed. By using Lemmas 9 and 10 and the properties of index, we have the following.

Theorem 2. Let $2<\alpha \leq 3,0<\eta<1, \beta \geq 0$, and $\gamma>0$ with $\gamma<((1+\beta) /(\eta+\beta))$. Suppose $f \in C^{1}([0, \infty), t[0, \infty))$. Assume that (H1) and (H2) hold. If $0 \leq f_{0}<(1 / r(L))$ and $(1 / r(L))<f_{\infty} \leq \infty$, then problem (4) has at least one positive solution on $[0,1]$.

Remark 5. Let $\beta=0, f_{0}=0$, and $f_{\infty}=\infty$; then, Theorem 2 reduces to Theorem 1.2 in [3].

By using Theorem 1, similar to the proof of Lemmas 8 and 9 as in [10], we can prove the following lemmas.

Lemma 11. Let $f \in C^{1}([0, \infty), t[0, \infty))$. Assume that $(H 1)$ and (H2) hold and $(1 / r(L))<f_{0} \leq \infty$. Then, there exists $\rho_{0}>0$ such that, for $\rho \in\left(0, \rho_{0}\right], i\left(T, P_{\rho}, P\right)=0$.

Lemma 12. Let $f \in C^{1}([0, \infty), t[0, \infty))$. Assume that $(H 1)$ and (H2) hold and $0 \leq f_{\infty}<(1 / r(L))$. Then, there exists $\tau_{0}>0$ such that, for $\tau>\tau_{0}, i\left(T, P_{\tau}, P\right)=1$.

By using Lemmas 11 and 12 and the properties of index, we have the following.

Theorem 3. Let $2<\alpha \leq 3,0<\eta<1, \beta \geq 0$, and $\gamma>0$ with $\gamma<((1+\beta) /(\eta+\beta))$. Suppose $f \in C^{1}([0, \infty), t[0, \infty))$. Assume that (H1) and (H2) hold. If $(1 / r(L))<f_{0} \leq \infty$ and $0 \leq f_{\infty}<(1 / r(L))$, then problem (4) has at least one positive solution on $[0,1]$.

Remark 6. Let $\beta=0, f_{0}=\infty$, and $f_{\infty}=0$; then, Theorem 3 reduces to Theorem 1.1 in [3].

\section{Examples}

In this section, two examples are given to illustrate the existence of positive solutions of BVP (4).

Example 1. Consider the following boundary value problem:

$$
\begin{cases}{ }^{C} D^{5 / 2} u(t)+t^{5 / 2} f(u(\theta(t)))=0, & 0<t<1, \\ u(0)-\frac{2}{3} u^{\prime}(0)=u^{\prime \prime}(0)=0, & u(1)=\frac{6}{5} u\left(\frac{1}{3}\right) .\end{cases}
$$

where $\alpha=5 / 2, \quad \beta=2 / 3, \quad \gamma=6 / 5, \quad \eta=1 / 3, \quad a(t)=t^{5 / 2}$, $\theta(t)=\sqrt{t}$, and

$$
f(u)= \begin{cases}3320 \sqrt{u^{3}}, & 0 \leq u \leq 1 \\ 3320(2 u-\sqrt{u}), & u>1\end{cases}
$$

Clearly, $f \in C^{1}([0, \infty), t[0, \infty))$. A direct computation shows

$$
\begin{aligned}
\omega & =\frac{\gamma \eta^{2-\alpha}(\alpha-1)(1-\eta)}{\Gamma(\alpha)(1+\beta-(\beta+\eta) \gamma)} \int_{0}^{\eta} a(s) s^{\alpha}(\eta-s)^{\alpha-2} \mathrm{~d} s \\
& =\frac{18 \sqrt{3}}{7 \Gamma(5 / 2)} \int_{0}^{1 / 3} s^{5}\left(\frac{1}{3}-s\right)^{1 / 2} \mathrm{~d} s \\
& =\frac{18 \sqrt{3}}{7 \Gamma(5 / 2)} \frac{\Gamma(6) \Gamma(3 / 2)}{\Gamma(15 / 2)}\left(\frac{1}{3}\right)^{(13 / 2)} \approx \frac{1}{6631} .
\end{aligned}
$$

Note that conditions (H1) and (H2) hold. Through a simple calculation, we can obtain $f_{0}=0$ and $f_{\infty}=6640>6631=(1 / \omega)>(1 / r(L))$ (see Lemma 8). Thus, by Theorem 2, we see that problem (43) has at least one positive solution.

Example 2. Consider the following boundary value problem:

$$
\begin{cases}{ }^{C} D^{8 / 3} u(t)+\sqrt{[3]} t^{4} f(u(\theta(t)))=0, & 0<t<1, \\ u(0)-\frac{1}{3} u^{\prime}(0)=u^{\prime \prime}(0)=0, & u(1)=\frac{3}{5} u\left(\frac{2}{3}\right) .\end{cases}
$$

Here, $\alpha=8 / 3, \beta=1 / 3, \gamma=3 / 5, \eta=2 / 3, a(t)=\sqrt{[3]} t^{4}$, $\theta(t)=\sqrt{[3]} t$, and

$$
f(u)= \begin{cases}93\left(\frac{2}{\pi^{2}} u^{2}+\frac{11}{2} \sin u\right), & 0 \leq u \leq \frac{\pi}{2} \\ 186\left(\sqrt{\frac{2}{\pi}} \sqrt{u}+2 \sin ^{2} u\right), & u>\frac{\pi}{2}\end{cases}
$$

Obviously, $f \in C^{1}([0, \infty), t[0, \infty))$. A direct computation shows 


$$
\begin{aligned}
\omega & =\frac{\gamma \eta^{2-\alpha}(\alpha-1)(1-\eta)}{\Gamma(\alpha)(1+\beta-(\beta+\eta) \gamma)} \int_{0}^{\eta} a(s) s^{\alpha}(\eta-s)^{\alpha-2} \mathrm{~d} s \\
& =\frac{5(3 / 2)^{2 / 3}}{11 \cdot \Gamma(8 / 3)} \int_{0}^{2 / 3} s^{4}\left(\frac{2}{3}-s\right)^{2 / 3} \mathrm{~d} s \\
& =\frac{5(3 / 2)^{2 / 3}}{11 \cdot \Gamma(8 / 3)} \frac{\Gamma(5) \Gamma(5 / 3)}{\Gamma(20 / 3)}\left(\frac{2}{3}\right)^{17 / 3} \approx 0.0022 .
\end{aligned}
$$

Note that conditions $(H 1)$ and $(H 2)$ hold. Obviously, we can get $f_{0}=511.5>454.5455=(1 / \omega)>(1 / r(L))$ and $f_{\infty}=0$. Thus, by Theorem 3 , we see that problem (46) has at least one positive solution.

\section{Data Availability}

No data were used to support this study.

\section{Conflicts of Interest}

The authors declare that they have no conflicts of interest.

\section{Acknowledgments}

This work was supported by the National Natural Science Foundation of China (no. 11571136).

\section{References}

[1] C. P. Gupta, "Solvability of a three-point nonlinear boundary value problem for a second order ordinary differential equation," Journal of Mathematical Analysis and Applications, vol. 168 , no. 2 , pp. 540-551, 1992.

[2] R. Ma, "Positive solutions of a nonlinear three-point boundary value problem," The Electronic Journal of Differential Equations, vol. 34, pp. 1-8, 1999.

[3] G. Wang, S. K. Ntouyas, and L. Zhang, "Positive solutions of the three-point boundary value problem for fractional-order differential equations with an advanced argument," Advances in Differential Equations, vol. 2011, no. 2, 2011.

[4] E. Pourhadi, R. Saadati, and S. K. Ntouyas, "Application of fixed-point theory for a nonlinear fractional three-point boundary value problem," Mathematics, vol. 2019, no. 7, 526 pages, 2019.

[5] J. R. L. Webb, "Positive solutions of some three point boundary value problems via fixed point index theory," Nonlinear Analysis: Theory, Methods and Applications, vol. 47, no. 7, pp. 4319-4332, 2001.

[6] X. Xu, "Multiplicity results for positive solutions of some semi-positone three-point boundary value problems," Journal of Mathematical Analysis and Applications, vol. 291, no. 2, pp. 673-689, 2004.

[7] B. Ahmad and J. J. Nieto, "Existence results for a coupled system of nonlinear fractional differential equations with three-point boundary conditions," Computers \& Mathematics with Applications, vol. 58, no. 9, pp. 1838-1843, 2009.

[8] H. Pang, M. Feng, and W. Ge, "Existence and monotone iteration of positive solutions for a three-point boundary value problem," Applied Mathematics Letters, vol. 21, no. 7, pp. 656-661, 2008.

[9] T. Jankowski, "Positive solutions for fourth-order differential equations with deviating arguments and integral boundary conditions," Nonlinear Analysis: Theory, Methods \& Applications, vol. 73, no. 5, pp. 1289-1299, 2010.

[10] B. Li, S. Sun, P. Zhao, and Z. Han, "Existence and multiplicity of positive solutions for a class of fractional differential equations with three-point boundary value conditions," Advances in Differential Equations, vol. 2015, no. 383, 2015.

[11] L. Peng and Y. Zhou, "Bifurcation from interval and positive solutions of the three-point boundary value problem for fractional differential equations," Applied Mathematics and Computation, vol. 257, pp. 458-466, 2015.

[12] P. D. Phung and L. X. Truong, "On the existence of a three point boundary value problem at resonance inRn," Journal of Mathematical Analysis and Applications, vol. 416, no. 2, pp. 522-533, 2014.

[13] X. Feng, H. Feng, and D. Bai, "Eigenvalue for a singular thirdorder three-point boundary value problem," Applied Mathematics and Computation, vol. 219, no. 18, pp. 9783-9790, 2013.

[14] S. K. Ntouyas and E. Pourhadi, "Positive solutions of nonlinear fractional three-point boundary-value problem," Le Mat, vol. 73, pp. 139-154, 2018.

[15] W. Sudsutad, J. Tariboon, and S. K. Ntouyas, "Positive solutions for fractional differential equations with three-point multi-term fractional integral boundary conditions," $\mathrm{Ad}$ vances in Differential Equations, vol. 2014, no. 28, 2014.

[16] J. Ren and W. Ge, "Positive solution for three-point boundary value problems with sign changing nonlinearities," Applied Mathematics Letters, vol. 17, no. 4, pp. 451-458, 2004.

[17] H.-Y. Li, "Existence of nontrivial solutions for superlinear three-point boundary value problems," Acta Mathematicae Applicatae Sinica, English Series, vol. 33, no. 4, pp. 1043-1052, 2017.

[18] Z. Bai and Y. Zhang, "Solvability of fractional three-point boundary value problems with nonlinear growth," Applied Mathematics and Computation, vol. 218, no. 5, pp. 1719-1725, 2011.

[19] J. Jiang, D. O’Regan, J. Xu, and X. Cui, "Positive solutions for a Hadamard fractional $p$-Laplacian three-point boundary value problem," Mathematics, vol. 2019, no. 7, 439 pages, 2019.

[20] D. Guo and V. Lakshmikantham, Nonlinear Problems in Abstract Cones, Academic Press, New York, NY, USA, 1988.

[21] A. A. Kilbas, H. M. Srivastava, and J. J. Trujillo, "Theory and applications of fractional differential equations," in North-Holland Mathematics Studies, vol. 204, Amsterdam, Netherlands, Elsevier, 2006.

[22] M. Cichon and A. H. Salem, "On the the lack of equivalent between differential and integral forms of the Caputo-type fractional problems," Journal of Pseudo-Differential Operators and Applications, vol. 11, pp. 1869-1895, 2020.

[23] E. Zeidler, Nonlinear Functional Analysis and Its Applications I: Fixed-Point Theorems, Springer, Berlin, Germany, 1985. 